\title{
Is COVID-19 Severity Impacted by Bariatric Surgery in the Early Postoperative Period?
}

\author{
Fernando Santa-Cruz ${ }^{1}$. Luciana T. Siqueira ${ }^{2}$. Lucas R. Coutinho ${ }^{3} \cdot$ Luís Henrique A. Leão $^{3}$. Amanda C. A. Almeida ${ }^{3}$. \\ Flávio Kreimer² · Álvaro A. B. Ferraz ${ }^{2}$
}

Received: 12 November 2021 / Revised: 4 January 2022 / Accepted: 14 January 2022 / Published online: 26 January 2022

(c) The Author(s), under exclusive licence to Springer Science+Business Media, LLC, part of Springer Nature 2022

\begin{abstract}
Purpose The aim of this study was to determine the real influence of bariatric surgery on the clinical evolution of patients infected with SARS-Cov-2 in the postoperative period.

Methods We conducted a retrospective analysis including two groups of patients: those who presented COVID-19 before bariatric surgery and those who presented it within 3 months of postoperative. Primary outcome was related to the severity of COVID-19, measured by the following variables: presence of symptoms, need for hospitalization, ICU admission, and invasive ventilation. Laboratory markers for inflammatory response, glycemic status, and micronutrients were analyzed as secondary outcomes.

Results From the 222 individuals operated on within the study period, only 66 (29.7\%) presented COVID-19, 42 (18.9\%) in the preoperative period and $24(10.8 \%)$ after the procedure. Mean age was $36.3 \pm 9.5$ years and mean preoperative BMI was $39.9 \pm 4.2 \mathrm{~kg} / \mathrm{m}^{2}$. There were no statistically significant differences between the groups regarding symptoms presentation $(92.9 \% \times 87.5 \%, p=0.66)$, need for hospitalization $(11.9 \% \times 16.7 \%, p=0.713)$, ICU admission $(4.8 \% \times 4.2 \%, p=1.000)$, and invasive ventilation $(2.4 \% \times 0.0 \%, p=1.000)$. Regarding the quantitative variables, absolute lymphocyte count was significantly lower in the group who presented COVID-19 after surgery $(1822.9 \pm 482.2 \times 2158.6 \pm 552.9, p=0.035)$.

Conclusion Patients who had COVID-19 before and after sleeve gastrectomy did not differ with statistical significance for the presence of symptoms, need for hospitalization, ICU admission, and invasive ventilation.
\end{abstract}

Keywords Bariatric surgery $\cdot$ Sleeve gastrectomy $\cdot$ Pandemic $\cdot$ COVID-19 $\cdot$ Sars-CoV-2

Key Points - Recent sleeve gastrectomy does not impact on symptoms presentation in COVID-19 patients

- Recent sleeve gastrectomy does not increase the need for hospitalization in COVID-19 patients

- Recent sleeve gastrectomy does not increase the need for invasive ventilation in COVID-19 patients

Álvaro A. B. Ferraz

alvaroabferraz@gmail.com

1 Department of Surgery, Post-Graduation in Surgery, Federal University of Pernambuco, Recife, PE, Brazil

2 Department of Surgery, Federal University of Pernambuco, Av. Prof. Moraes Rego, 1235, Cidade Universitária, Recife, PE 50670-901, Brazil

3 Medical School, Federal University of Pernambuco, Recife, PE, Brazil

\section{Introduction}

Infection caused by SARS-CoV-2 has been considered a pandemic since the beginning of 2020, and approximately $4,517,240$ deaths have been registered to date $[1,2]$. Brazil was ranked third in the number of confirmed cases of COVID-19 and second in the number of deaths from the disease [3].

In view of the high risk of infection and the pressure on health services, various spheres of elective medical care suspended activities amidst the outbreak of the pandemic. Biosafety measures imposed during the outbreak of COVID19 mostly affected elective surgeries, which account for about $91 \%$ of the procedures in the USA [4].

In the case of bariatric surgery, frequent delays and/or cancellations of the procedure during this period can seriously affect the quality of life and the preoperative preparation of eligible patients $[5,6]$. The delay in performing the 
procedure perpetuates baseline conditions of the patient, such as diabetes and obesity, which are considered important risk factors for severe cases of COVID-19 [5, 7-10]. On the other hand, the hypercatabolic state and immune depression recorded in the postoperative period of bariatric surgery are factors of concern for worse clinical outcomes in case of SARS-Cov-2 infection [11, 12]. This dichotomy raises important questions about the risk-benefit ratio of bariatric surgery during the current pandemic. To date, there is no consensus on this issue in the literature.

In this scenario, the present study analyzes the safety of performing bariatric surgeries during the COVID-19 pandemic. For this purpose, we assessed the influence of this procedure on the clinical evolution of patients infected with SARS-Cov-2 in the postoperative period.

\section{Methods}

\section{Study Design}

A retrospective cohort study conducted from June 2021 to September 2021 that analyzed all patients undergoing bariatric surgery at our center during the period between April 2020 and May 2021, who presented pre- or postoperative Sars-CoV-2 infection. The surgical technique performed was sleeve gastrectomy (SG).

All procedures performed in this study involving human participants were in accordance with the ethical standards of the institutional research committee and the 1964 Helsinki Declaration and its later amendments. This research project was approved by the Ethics Committee of our institution under the protocol CAAE 48,206,421.1.0000.8807.

\section{Study Population}

The sampling method was non-probabilistic, selected through convenience. The study included all patients aged between 18 and 60 years of both genders, with body mass index $(\mathrm{BMI}) \geq 35 \mathrm{~kg} / \mathrm{m}^{2}$, who underwent bariatric surgery during the current pandemic period in our institution and who developed COVID-19 in either the pre- or postoperative period. Patients at high cardiovascular risk, patients requiring intensive postoperative care, and patients with a $\mathrm{BMI} \geq 50 \mathrm{~kg} / \mathrm{m}^{2}$ were excluded from the final analysis.

\section{Data Collection}

The data were collected through an electronic database. Patients were divided into two groups: preoperative COVID-19 versus postoperative COVID-19. The primary outcome was the severity of viral infection in the patients, translated into the following categorical variables: presence of symptoms, need for hospitalization, intensive care unit (ICU) admission, and invasive ventilation (orotracheal intubation). Secondary outcomes consisted of quantitative variables regarding the glycemic status (fasting glucose, HbAlc, serum insulin, C-peptide), serum dosage of micronutrients (zinc, vitamins D and B12, calcium, and iron), and systemic inflammation markers (leukocytes, lymphocytes, C-reactive protein, ferritin, albumin). We compared the groups at the two assessment times (preoperatively and 3 months postoperatively). All cases of COVID-19 were confirmed by RTPCR at the time of illness.

No patient was operated on with active viral infection. Every patient collected RT-PCR for COVID-19 within the 3 days preceding the surgery schedule. If the test was positive, patients required quarantine at home, surgery would be postponed 10 days and a new RT-PCR collected. After discharge, patients were instructed to collect new RT-PCR samples if COVID-19 symptoms occur. For those patients who presented symptomatic COVID-19 during the preoperative period, surgery would be re-scheduled within, at least, 7 weeks. The vaccine status was not assessed since the study was initiated before the release of the first samples in Brazil.

\section{Statistical Analysis}

Data were descriptively analyzed using absolute frequencies and percentages for categorical variables and mean and standard deviation for numerical variables. To assess differences between the groups in relation to categorical variables, we used the chi-square test or Fisher's exact test, Student's T test with equal variances, Student's T test with unequal variances or Mann-Whitney test. The Student's T test was chosen in situations where the data did not present a normal distribution, and the Mann-Whitney test in case of lack of normality. Normality was verified by the Shapiro-Wilk test and equality of variances by the Levene's F test. The analysis of each group in relation to categorical variables provided the relative risk and respective confidence interval. The level of significance in the statistical tests was $95 \%$. Data were entered into an Excel ${ }^{\circledR}$ spreadsheet and the program SPSS (version 25) was used to obtain statistical calculations.

\section{Results}

A total of 222 individuals were operated on within the study period. However, only 66 individuals were included in the final analysis: 42 in the group with preoperative COVID-19 and 24 in the group with postoperative COVID-19. Mean age was $36.3 \pm 9.5$ years, mean preoperative BMI was $39.9 \pm 4.2 \mathrm{~kg} / \mathrm{m}^{2}$, and mean postoperative BMI (3 months) was $32.3 \pm 3.8 \mathrm{~kg} / \mathrm{m}^{2}$. The overall incidence of COVID-19 in the total population was $29.7 \%$, occurring preoperatively 
in $18.9 \%$ of the patients and postoperatively in $10.8 \%$. Only $7.6 \%$ of the population had a preoperative diagnosis of diabetes (Table 1).

Table 2 shows data regarding the severity of the clinical condition of COVID-19 before and after bariatric surgery (primary outcome). None of the variables under study differed with statistical significance between the groups. In the entire series, only 1 patient required orotracheal intubation. All cases of hospital and ICU admissions were due to COVID-19 symptomatology.

Table 1 Demographic data

\begin{tabular}{ll}
\hline Women (\%) & $168(75.7)$ \\
\hline Preoperative COVID-19 & $32(76.2)$ \\
Postoperative COVID-19 & $19(79.2)$ \\
Age (years) & $36.3 \pm 9.5$ \\
Preoperative COVID-19 & $37.0 \pm 8.3$ \\
Postoperative COVID-19 & $34.9 \pm 11.7$ \\
$p$-value & 0.22 \\
Preoperative BMI (Kg/m $\left.{ }^{2}\right)$ & $39.9 \pm 4.2$ \\
Preoperative COVID-19 & $39.4 \pm 3.9$ \\
Postoperative COVID-19 & $41.0 \pm 4.6$ \\
$p$-value & 0.07 \\
Postoperative BMI (Kg/m ${ }^{2}$ ) & $32.3 \pm 3.8$ \\
Preoperative COVID-19 & $31.8 \pm 3.7$ \\
Postoperative COVID-19 & $33.3 \pm 4.1$ \\
$p$-value & 0.09 \\
Overall incidence of COVID-19 (\%) & 29.7 \\
Overall incidence of preoperative COVID-19 (\%) & 18.9 \\
Overall incidence of postoperative COVID-19 (\%) & 10.8 \\
Diabetes (\%) & $17(7.6)$ \\
\hline
\end{tabular}

Table 3 shows the preoperative results of laboratory parameters between the groups. During this period, the groups did not differ with statistical significance for the markers of inflammation/immunity, glycemic profile, and micronutrients.

Table 4 shows the laboratory parameters measured 3 months after the surgical procedure. Among all the variables under study, only the total lymphocyte count differed between the groups, being significantly lower in the group that presented COVID-19 after bariatric surgery. The other variables were equivalent between groups.

\section{Discussion}

Bariatric surgery is the main choice in the treatment of obesity and its comorbidities, risk factors that account for worse clinical outcomes in COVID-19. However, there is still no clear consensus on the safety of this procedure in the context of the current pandemic. In a first analysis, the potential resolution of the aforementioned risk factors seems to endorse the performance of these surgeries. Notwithstanding, the immunological dysfunction and the hypercatabolic state inherent to the endocrine and metabolic response to surgical trauma emerge as important counterpoints to be analyzed in the current context [11,12].

A multicenter cross-sectional study assessed the clinical outcomes of COVID-19 in the pre- and postoperative period of bariatric surgery. In that study, the authors compared the occurrence and severity of SARS-CoV-2 infection between patients submitted to bariatric surgery and patients candidates for this procedure [13]. In line with our results, the frequency of hospitalization due to COVID19 did not differ with statistical significance between the
Table 2 Assessment of the severity of COVID-19 among the groups under study

\begin{tabular}{|c|c|c|c|c|}
\hline \multirow[b]{2}{*}{ Variable } & \multicolumn{2}{|l|}{ COVID-19 } & \multirow[b]{2}{*}{$P$ value } & \multirow[b]{2}{*}{$R R(C I 95 \%)$} \\
\hline & Preoperative (42) & Postoperative (24) & & \\
\hline & $n(\%)$ & $n(\%)$ & & \\
\hline Symptoms & & & $p^{(1)}=0.660$ & \\
\hline Yes & $39(92.9)$ & $21(87.5)$ & & $1.1(0.9$ to 1.4$)$ \\
\hline No & $3(7.1)$ & $3(12.5)$ & & \\
\hline Hospitalization & & & $p^{(1)}=0.713$ & \\
\hline Yes & $5(11.9)$ & $4(16.7)$ & & $1.4(0.4$ to 4.7$)$ \\
\hline No & $37(88.1)$ & $20(83.3)$ & & \\
\hline ICU & & & $p^{(1)}=1.000$ & \\
\hline Yes & $2(4.8)$ & $1(4.2)$ & & $1.1(0.1$ to 11.9$)$ \\
\hline No & $40(95.2)$ & $23(95.8)$ & & \\
\hline Invasive ventilation & & & $p^{(1)}=1.00$ & \\
\hline Yes & $1(2.4)$ & - & & $*$ \\
\hline No & $41(97.6)$ & $24(100.0)$ & & $*$ \\
\hline
\end{tabular}

(*) Null frequency, (1) Fisher's exact test 
Table 3 Biochemical data in the preoperative assessment

\begin{tabular}{|c|c|c|c|}
\hline & \multicolumn{2}{|l|}{ Preoperative assessment } & \\
\hline & Preoperative COVID-19 & Postoperative COVID-19 & \\
\hline Variable & Mean \pm SD & Mean \pm SD & $P$ value \\
\hline Leukocytes $\left(\right.$ cells $/ \mathrm{mm}^{3}$ ) & $6748.43 \pm 1176.77$ & $7298.70 \pm 1736.16$ & $p^{(1)}=0.299$ \\
\hline Lymphocytes (cells $\left./ \mathrm{mm}^{3}\right)$ & $2241.19 \pm 604.38$ & $2092.63 \pm 473.92$ & $p^{(2)}=0.305$ \\
\hline Platelets (cells $/ \mathrm{mm}^{3}$ ) & $284,154.76 \pm 79,828.93$ & $282,291.67 \pm 55,484.80$ & $p^{(1)}=0.856$ \\
\hline Fasting plasma glucose (mg/dL) & $101.06 \pm 13.58$ & $105.03 \pm 24.10$ & $p^{(1)}=0.934$ \\
\hline C-reactive protein $(\mathrm{mg} / \mathrm{L})$ & $4.34 \pm 6.13$ & $4.31 \pm 5.38$ & $p^{(1)}=0.809$ \\
\hline $\operatorname{HbA} 1 \mathrm{C}(\%)$ & $5.73 \pm 0.46$ & $5.83 \pm 0.93$ & $p^{(1)}=0.579$ \\
\hline Insulin (mU/L) & $31.72 \pm 44.36$ & $27.00 \pm 13.92$ & $p^{(1)}=0.753$ \\
\hline C-Peptide (ng/mL) & $3.72 \pm 1.36$ & $3.82 \pm 1.42$ & $p^{(1)}=0.549$ \\
\hline Calcium (mg/dL) & $11.23 \pm 13.72$ & $9.07 \pm 0.49$ & $p^{(1)}=0.375$ \\
\hline Zinc (mcg/dL) & $82.81 \pm 22.41$ & $91.11 \pm 13.78$ & $p^{(1)}=0.108$ \\
\hline Vitamin B12 (pg/mL) & $455.50 \pm 153.24$ & $439.92 \pm 150.58$ & $\mathrm{p}^{(2)}=0.691$ \\
\hline Iron $(\mathrm{mcg} / \mathrm{dL})$ & $81.05 \pm 28.91$ & $79.24 \pm 31.86$ & $p^{(1)}=0.590$ \\
\hline Ferritin $(\mathrm{ng} / \mathrm{mL})$ & $178.35 \pm 202.11$ & $221.82 \pm 371.79$ & $p^{(1)}=0.684$ \\
\hline Albumin $(\mathrm{g} / \mathrm{L})$ & $4.39 \pm 0.31$ & $4.27 \pm 0.37$ & $p^{(2)}=0.184$ \\
\hline Vitamin D (ng/mL) & $24.05 \pm 10.14$ & $23.29 \pm 7.09$ & $p^{(1)}=0.839$ \\
\hline
\end{tabular}

(*) Significant difference at 5.0\%; (1) Mann-Whitney test; (2) Student's $t$-test with equal variances

\begin{tabular}{|c|c|c|c|}
\hline & \multicolumn{2}{|l|}{ Assessment after 3 months } & \\
\hline & Preoperative COVID-19 & Postoperative COVID-19 & \\
\hline Variable & Mean \pm SD & Mean \pm SD & $P$ value \\
\hline Leukocytes (cells/mm³) & $5751.85 \pm 1555.99$ & $5579.00 \pm 1504.68$ & $p^{(1)}=0.869$ \\
\hline Lymphocytes (cells/mm³) & $2158.63 \pm 552.95$ & $1822.90 \pm 482.21$ & $p^{(2)}=0.035^{*}$ \\
\hline Platelets (cells $/ \mathrm{mm}^{3}$ ) & $250,622.22 \pm 78,892.77$ & $245,835.00 \pm 102,312.19$ & $p^{(1)}=0.705$ \\
\hline Fasting plasma glucose (mg/dL) & $85.04 \pm 7.41$ & $86.27 \pm 7.95$ & $p^{(2)}=0.591$ \\
\hline C-reactive protein $(\mathrm{mg} / \mathrm{L})$ & $2.13 \pm 3.64$ & $1.94 \pm 2.58$ & $p^{(1)}=0.372$ \\
\hline $\operatorname{HbA} 1 \mathrm{C}(\%)$ & $5.29 \pm 0.37$ & $5.41 \pm 0.53$ & $p^{(1)}=0.826$ \\
\hline Insulin (mU/L) & $10.45 \pm 4.82$ & $10.67 \pm 5.40$ & $p^{(1)}=0.936$ \\
\hline C-peptide (ng/mL) & $2.37 \pm 0.78$ & $2.32 \pm 0.84$ & $p^{(2)}=0.812$ \\
\hline Calcium (mg/dL) & $9.11 \pm 0.49$ & $9.19 \pm 0.39$ & $p^{(2)}=0.528$ \\
\hline Zinc (mcg/dL) & $111.19 \pm 172.53$ & $88.69 \pm 15.12$ & $p^{(2)}=0.173$ \\
\hline Vitamin B12 (pg/mL) & $577.25 \pm 316.28$ & $687.40 \pm 386.25$ & $p^{(1)}=0.241$ \\
\hline Iron $(\mathrm{mcg} / \mathrm{dL})$ & $81.59 \pm 24.54$ & $76.22 \pm 21.01$ & $p^{(1)}=0.372$ \\
\hline Ferritin (ng/mL) & $170.54 \pm 118.88$ & $178.04 \pm 188.47$ & $p^{(1)}=0.605$ \\
\hline Albumin $(\mathrm{g} / \mathrm{L})$ & $4.34 \pm 0.33$ & $4.26 \pm 0.30$ & $p^{(1)}=0.437$ \\
\hline Vitamin D (ng/mL) & $29.88 \pm 8.50$ & $31.28 \pm 5.85$ & $p^{(2)}=0.530$ \\
\hline
\end{tabular}

(*) Significant difference at 5.0\%; (1) Mann-Whitney test; (2) Student's $t$-test with equal variances
Table 4 Biochemical data in the postoperative assessment (3 months after surgery) groups $(2.4 \% \times 0.3 \%, p>0.05)$. Regarding admission to the ICU, on the other hand, no participant in the study in question had such an outcome, while our data showed an admission rate of $4.2 \%$ and $4.8 \%$ for cases with infected patients in the preoperative and postoperative periods, respectively $(p>0.05)$. However, it is important to highlight that, despite the aforementioned study having a larger sample, only $5.7 \%$ of the participants had a confirmed diagnosis of COVID-19 [13]. This may explain the differences in relation to the data of the present study.

Data from the COVIDSurg Collaborative [14] point to pulmonary complications in $51.2 \%$ of patients undergoing elective or emergency surgery who tested positive for COVID-19 7 days before or up to 30 days after the procedure, with a 30 -day mortality rate of $23.8 \%$. In the scope of elective surgeries, $52.5 \%$ of patients had pulmonary 
complications such as pneumonia, acute respiratory distress syndrome, and need for invasive ventilation. The latter occurred in $14.2 \%$ of patients, and the 30-day mortality rate in the postoperative period of elective surgeries was $18.9 \%$ [14].

In contrast to these data, our study showed significantly lower rates of hospital admission, ICU admission, and need for invasive ventilation in patients with COVID-19 in the postoperative period of bariatric surgery. This difference may be due to the study periods, as the COVIDSurg Collaborative took place at the beginning of the COVID-19 pandemic, corresponding to a period of development of surgical protocols in case of perioperative infection by SARS-CoV-2 [14].

The homogeneity evidenced in the analysis of variables regarding the severity of SARS-CoV-2 infection in pre- and postoperative moments in the present study encourages the performance of bariatric surgery during the current pandemic, since the procedure did not worsen clinical outcomes. In line with these results, the GENEVA study [15] evaluated the performance of 2001 bariatric surgeries between May and July 2020, addressing the complication rate 30 days after the procedure. Of the total number of patients analyzed, only $10(0.5 \%)$ tested positive for COVID-19 during the analysis period. Of these, none required admission to the ICU or invasive ventilation. In addition, the study demonstrated 30 -day mortality rates of $0.05 \%$, similar to those occurring in prepandemic periods [15].

The data obtained in the GENEVA study were further complemented by a multinational cohort comprising 7704 patients analyzed between May and October 2020 [16]. Of the total number of patients, $43(0.56 \%)$ had symptomatic COVID-19 in the first 30 days after surgery. Of these, $74.4 \%$ were operated on in hospitals also used for the treatment of COVID-19. Most of these patients had surgical complications Clavien-Dindo I (67.4\%) or II (25.6\%), which may be due to the longer postoperative hospital stay. The study reported only one death related to COVID-19 [16].

In the current scenario of pandemic decline and relaxation of restrictive measures, bariatric surgery centers seem to gradually return to the pre-SARS-CoV-2 status, but there is still no consensus on the safety of the procedure in candidates recovered from COVID-19 [17]. Nedelcu et al. aimed to provide evidence for such questioning through a multicentric cohort in which the authors described the clinical pictures of 35 individuals with severe obesity and documented previous infection by SARS-CoV-2, also analyzing their postoperative complications [18]. In comparison to the clinical evolution of patients in the preoperative group in our study, participants in that study had considerably fewer symptoms related to COVID-19 (42.9\% vs $92.9 \%)$. However, this fact did not correlate with the severity of infection since the hospitalization rate $(11.9 \%$ vs $14.3 \%)$ and the need for ICU admission (4.8\% vs $2.86 \%)$ were similar between the analyses.

It is also worth mentioning the 30-day follow-up after bariatric surgery by the aforementioned reference, which demonstrated the occurrence of 2 readmissions and only one case of surgical complication (gastric leak treated with laparoscopic drainage) 18. In association with our data and those of the existing literature, these results seem to endorse the safety of performing bariatric surgeries in the context of the SARS-CoV-2 pandemic [18, 19].

The present study has some limitations that deserve discussion. The first regards the observational and retrospective nature of the analysis, which limits the ability of the study to confirm the raised hypothesis. Moreover, the sample size was reduced, as only confirmed cases of COVID-19 were included in the final analysis.

\section{Conclusion}

Patients who had COVID-19 before and after sleeve gastrectomy did not differ with statistical significance for presence of symptoms, need for hospitalization, need for ICU admission, and need for orotracheal intubation (invasive ventilation). The results of the present study thus raise the hypothesis that bariatric surgery is safe to be performed during the pandemic period, as it does not interfere with the course of Sars-CoV-2 infection in the first 3 months after surgery. Further studies are needed involving a larger number of patients, with greater control of preoperative comorbidities, and having a multicenter nature. These should focus on determining the safety profile of this procedure during the current pandemic period.

Acknowledgements The authors gratefully acknowledge the members of the General Surgery Division, Hospital das Clínicas, Federal University of Pernambuco (HC/UFPE - EBSERH) for all the support provided to this research and the whole Bariatric Surgery Program.

\section{Declarations}

Ethics Approval All procedures performed in this study involving human participants were in accordance with the ethical standards of the institutional research committee and the 1964 Helsinki Declaration and its later amendments. This research project was approved by the Ethics Committee of our institution under the protocol CAAE 48206421.1.0000.8807.

Consent to Participate Informed consent does not apply.

Conflict of Interest The authors declare no competing interests. 


\section{References}

1. World Health Organization. WHO Director-General's opening remarks at the media briefing on COVID-19; 2020. Available at: https://www.who.int/director-general/speeches/detail/who-direc tor-general-s-opening-remarks-at-the-media-briefing-on-covid19---11-march-2020. Accessed September $2^{\text {nd }}, 2021$.

2. World Health Organization. WHO Coronavirus (COVID-19) Dashboard. Avalable at: https://covid19.who.int. Accessed September $2^{\text {nd }}, 2021$.

3. Marcolino MS, Ziegelmann PK, Souza-Silva MVR, et al. Clinical characteristics and outcomes of patients hospitalized with COVID-19 in Brazil: results from the Brazilian COVID-19 registry. Int J Infect Dis. 2021;107:300-10.

4. Prin M, Guglielminotti J, Mtalimanja O, Li G, Charles A. Emergency-to-elective surgery ratio: a global indicator of access to surgical care. World J Surg. 2018;42(7):1971-80.

5. Fu SJ, George EL, Maggio PM, Hawn M, Nazerali R. The consequences of delaying elective surgery: surgical perspective. Ann Surg. 2020;272(2):e79-80.

6. Hussain A, Mahawar K, El-Hasani S. The impact of COVID19 pandemic on obesity and bariatric surgery. Obes Surg. 2020;30(8):3222-3.

7. Marhl M, Grubelnik V, Magdič M, Markovič R. Diabetes and metabolic syndrome as risk factors for COVID-19. Diabetes Metab Syndr. 2020;14(4):671-7.

8. Williamson EJ, Walker AJ, Bhaskaran K, et al. Factors associated with COVID-19-related death using OpenSAFELY. Nature. 2020;584(7821):430-6.

9. Hendren NS, de Lemos JA, Ayers C, et al. Association of body mass index and age with morbidity and mortality in patients hospitalized with COVID-19: results from the American Heart Association COVID-19 cardiovascular disease registry. Circulation. 2021;143(2):135-44.

10. Cottini M, Lombardi C, Berti A, et al. Obesity is a major risk factor for hospitalization in community-managed COVID-19 pneumonia. Mayo Clin Proc. 2021;96(4):921-31.
11. Lord JM, Midwinter MJ, Chen YF, et al. The systemic immune response to trauma: an overview of pathophysiology and treatment. Lancet. 2014;384(9952):1455-65.

12. Menges P, Kessler W, Kloecker C, et al. Surgical trauma and postoperative immune dysfunction. Eur Surg Res. 2012;48(4):180-6.

13. Marchesi F, Valente M, Riccò M, et al. Effects of bariatric surgery on COVID-19: a multicentric study from a high incidence area. Obes Surg. 2021;31(6):2477-88.

14. Nepogodiev D, Glasbey JC, Li E, et al. Mortality and pulmonary complications in patients undergoing surgery with perioperative SARS-CoV-2 infection: an international cohort study. The Lancet. 2020.; 396(10243): 27-38.

15. Singhal R, Tahrani AA, Ludwig C, et al. Global 30-day outcomes after bariatric surgery during the COVID-19 pandemic (GENEVA): an international cohort study. Lancet Diabetes Endocrinol. 2021;9(1):7-9.

16. Singhal R, Ludwig C, Rudge G, et al. 30-day morbidity and mortality of bariatric surgery during the COVID-19 pandemic: a multinational cohort study of 7704 patients from 42 countries. Obes Surg. 2021;31(10):4272-88.

17. Rubino F, Cohen RV, Mingrone G, et al. Bariatric and metabolic surgery during and after the COVID-19 pandemic: DSS recommendations for management of surgical candidates and postoperative patients and prioritisation of access to surgery. Lancet Diabetes Endocrinol. 2020;8(7):640-8.

18. Nedelcu M, Marx L, Lutfi RE, et al. Bariatric surgery in patients with previous COVID-19 infection. Surg Obes Relat Dis. 2021;17(7):1244-8.

19. Vosburg RW, Pratt JSA, Kindel T, et al. Bariatric Surgery is safe for patients after recovery from COVID-19. Surg Obes Relat Dis. 2021;17(11):1884-9.

Publisher's Note Springer Nature remains neutral with regard to jurisdictional claims in published maps and institutional affiliations. 